PROCEEDINGS OF THE

AMERICAN MATHEMATICAL SOCIETY

Volume 127, Number 7, Pages 1967-1971

S 0002-9939(99)04758-9

Article electronically published on March 16, 1999

\title{
HÖLDER ABSOLUTE VALUES ARE EQUIVALENT TO CLASSICAL ONES
}

\author{
E. MUÑOZ GARCIA
}

(Communicated by Wolmer V. Vasconcelos)

\begin{abstract}
We study generalized absolute values on a field or a commutative ring with unit element satisfying an approximate triangle inequality and an approximate multiplicative property. We prove that they are always Hölder equivalent to an absolute value. This implies geometric rigidity results for Lipschitz and Hölder deformations of metric rings.
\end{abstract}

\section{INTRODUCTION}

We define in this article Hölder absolute values, which are a generalization of the classical notion of absolute value on a field. Hölder absolute values satisfy an approximate triangle inequality and an approximate multiplicative property.

More precisely, let $C_{1} \geq 1$ and $C_{2} \geq 1$. A $\left(C_{1}, C_{2}\right)$-Hölder absolute value on a ring $R$ is a mapping $\|\|:. R \rightarrow \mathbf{R}_{+}$satisfying:

(HAV1) For $x \in R,\|x\|=0 \Leftrightarrow x=0$.

(HAV2) For $x, y \in R,\|x+y\| \leq C_{2}(\|x\|+\|y\|)$.

(HAV3) For $x, y \in R, C_{1}^{-1}\|x\|\|y\| \leq\|x y\| \leq C_{1}\|x\|\|y\|$.

Note that a ring which admits a Hölder absolute value is necessarily a ring without zero divisors. We note that a classical absolute value is a (1,1)-Hölder absolute value. Our theorem shows that any Hölder absolute value on a division ring or a commutative ring with unit element is Hölder equivalent to a classical one. We recall that two metric structures $(X, d)$ and $(X, \delta)$ are $\left(C_{0}, \alpha\right)$-Hölder equivalent, $C_{0} \geq 1, \alpha>0$, if for $x, y \in X$,

$$
C_{0}^{-1} d(x, y)^{\alpha^{\prime}} \leq \delta(x, y) \leq C_{0} d(x, y)^{\alpha^{\prime}},
$$

where $\alpha^{\prime}=a$ or $\alpha^{\prime}=a^{-1}$.

Theorem (Hölder Rigidity). Let $\|\|:. R \rightarrow \mathbf{R}_{+}$be a $\left(C_{1}, C_{2}\right)$-Hölder absolute value on a division ring or a commutative ring $R$ with unit element. There exists an absolute value on $R,||:. R \rightarrow \mathbf{R}_{+}$, which is $\left(C_{1}^{\alpha}, \alpha\right)$-Hölder equivalent to $\|$. with $\alpha=\log _{2}\left(2 C_{2}\right)$, i.e. for $x \in R$,

$$
C_{1}^{-\alpha}|x|^{\alpha} \leq\|x\| \leq C_{1}^{\alpha}|x|^{\alpha} .
$$

Received by the editors June 25, 1997 and, in revised form, October 14, 1997.

1991 Mathematics Subject Classification. Primary 12J20; Secondary 12J10, 16W80, 13J99.

Key words and phrases. Valuations, Ostrowski's Theorem, Hölder deformations, metric spaces.

(C)1999 American Mathematical Society 
Moreover |.| can be defined by

$$
|x|=\lim _{n \rightarrow+\infty}|| x^{n}||^{\frac{1}{n \alpha}} .
$$

N. Bourbaki has studied $\left(1, C_{2}\right)$-Hölder absolute values ([Bou]). Our Theorem generalizes Bourbaki's result (let $C_{1}=1$ ):

Corollary (Bourbaki). If $\|$.$\| is a \left(1, C_{2}\right)$-Hölder absolute value over a field, then there exists an absolute value $|$.$| and an exponent \alpha$ such that $\|x\|=|x|^{\alpha}$.

Over $\mathbf{Q}$ this result can be seen as a quantitative generalization of Ostrowski's Theorem for absolute values $\left(C_{1}=1\right.$ and $C_{2}=1$ give Ostrowski's Theorem).

Corollary. If $\|$.$\| is a \left(C_{1}, C_{2}\right)$-Hölder absolute value over $\mathbf{Q}$, normalized so that $\|1\|=1$, then $\|$.$\| is \left(C_{1}^{\alpha}, \alpha\right)$-Hölder equivalent to a $p$-adic absolute value $|\cdot|_{p}$ or to $|\cdot|_{\infty}$, with $\alpha=\log _{2}\left(2 C_{2}\right)$.

Our main motivation is the study of geometric deformations of metric rings. The notion of Hölder absolute value appears naturally considering Hölder deformations of the metric space defined by a ring with an absolute value such that all translations are uniformly Lipschitz in the new metric. This explains the terminology used. In this geometric setting our Theorem can be formulated as a rigidity result for Lipschitz and Hölder deformations of metric rings.

The proof of the main theorem consists of two parts. First we prove that the limit defining |.| always exists. Second we show that |.| is an absolute value. The triangle inequality is the tricky part.

Proof of the theorem. Let $R$ be a division ring or a commutative ring with unit element endowed with a $\left(C_{1}, C_{2}\right)$-Hölder absolute value, denoted by $\|$.$\| , with C_{1} \geq$ 1 and $C_{2} \geq 1$.

Taking $\alpha=\frac{1}{\log _{2}\left(2 C_{2}\right)}$, we have for any $x, y \in R$,

$$
\begin{aligned}
\|x+y\|^{\alpha} & \leq\left(2 C_{2}\right)^{\alpha}(\max (\|x\|,\|y\|))^{\alpha} \\
& \leq 2 \max \left(\|x\|^{\alpha},\|y\|^{\alpha}\right) .
\end{aligned}
$$

Define the mapping $\|||\|:. R \rightarrow \mathbf{R}_{+}$by $\||x|\|=\|x\|^{\alpha}$. Obviously, we have:

Lemma 1. The map $\|\mid\| \cdot \|$ is a $\left(C_{1}^{\alpha}, 2\right)$-Hölder absolute value on $R$.

Consider $x \in R, x \neq 0$, and define the sequence of positive numbers $\left(a_{n}\right)_{n \geq 0}$ by $a_{n}=C_{1}^{\alpha}||\left|x^{n}\right|||$. The following Lemmata are straightforward:

Lemma 2. The sequence $\left(a_{n}\right)_{n \geq 0}$ is sub-multiplicative, i.e. for $n, m \geq 1, a_{n m} \leq$ $a_{n} a_{m}$.

Lemma 3. We have $C_{1}^{-\alpha}|||x|\left\|\leq\left|\left\|\left.x^{n}||\right|^{1 / n} \leq C_{1}^{\alpha}|\|x|\||\right.\right.\right.$.

It is a classical exercise that Lemma 2 implies $\lim \inf a_{n}^{1 / n}=\lim \sup a_{n}^{1 / n}$. Moreover Lemma 3 implies that the sequence $\left(a_{n}^{1 / n}\right)_{n \geq 0}$ is bounded above and bounded away from 0 . So this sequence has a limit

$$
|x|=\lim _{n \rightarrow+\infty} a_{n}^{1 / n}=\lim _{n \rightarrow+\infty}||\left|x^{n}\right| \|^{1 / n} .
$$

We also define $|0|=0$. Using Lemma 3 we prove: 
Proposition 1. For $x \in R, C_{1}^{-\alpha}|||x| \||\leq| x\left|\leq C_{1}^{\alpha}\right||| x|| \mid$.

So we only need to prove that |.| is indeed an absolute value. The only delicate part is proving the triangle inequality.

Lemma 4. (AV1) For $x \in R,|x|=0 \Leftrightarrow x=0$.

(AV3) For $x, y \in R,|x y|=|x||y|$.

Proof. (AV1) follows from Proposition 1.

For $x, y \in R$, we have

$$
C_{1}^{-\alpha}||\left|x^{n}\right|||\left\|y^{n}|||\leq||| x^{n} y^{n}||\left|\leq C_{1}^{\alpha}\right||| x^{n}||||\left|y^{n}\right|\right\| .
$$

Taking $n$ roots and passing to the limit $n \rightarrow+\infty$ we get

$$
|x||y| \leq|x y| \leq|x||y|,
$$

so we have the equality and (AV3).

So |.| has the expected multiplicative behavior. At first we only have an approximate version of the triangle inequality (AV2).

Lemma 5. For $x, y \in R$ we have

$$
|x+y| \leq C_{1}^{2 \alpha}(|x|+|y|) \leq 2 C_{1}^{2 \alpha} \max (|x|,|y|) .
$$

Proof. We use Proposition 1 twice and the triangle inequality for $\||| .||$ :

$$
|x+y| \leq C_{1}^{\alpha}|||x+y| \| \leq C_{1}^{\alpha}(|||x| \||+||| y|| \mid) \leq C_{1}^{2 \alpha}(|x|+|y|) .
$$

Because of the previous approximate triangle inequality it is much more efficient to cut sums dyadically. The next lemma and its proof recalls the algorithmic problem of sorting a finite set of integers (the complexity of the naïve algorithm is $\mathcal{O}\left(n^{2}\right)$ but the dyadic optimal algorithm has complexity $\left.\mathcal{O}(n \log n)\right)$; see for example [We], p. 137).

Lemma 6 (Dyadic trick). Let $|\cdot|^{\prime}: R \rightarrow \mathbf{R}_{+}$such that for $x, y \in R$ we have

$$
|x+y|^{\prime} \leq M \max \left(|x|^{\prime},|y|^{\prime}\right),
$$

for some positive constant $M$. Then for $x_{1}, x_{2}, \ldots, x_{n} \in R$, we have

$$
\left|\sum_{i=1}^{n} x_{i}\right|^{\prime} \leq M^{\left[\log _{2} n\right]+1} \max _{1 \leq i \leq n}\left(\left|x_{i}\right|^{\prime}\right),
$$

where $[a]$ denotes the integer part of $a$.

Proof. Let $m=\left[\log _{2} n\right]+1$ and complete the sequence $\left(x_{i}\right)_{1 \leq i \leq n}$ into $\left(x_{i}\right)_{1 \leq i \leq 2^{m}}$ adjoining 0 elements. Then using the approximate triangle inequality:

$$
\begin{aligned}
\left|\sum_{i=1}^{2^{m}} x_{i}\right|^{\prime} & \leq M \max \left(\left|\sum_{i=1}^{2^{m-1}} x_{i}\right|^{\prime},\left|\sum_{i=2^{m-1}+1}^{2^{m}} x_{i}\right|^{\prime}\right) \\
& \leq M^{2} \max \left(\left|\sum_{i=1}^{2^{m-2}} x_{i}\right|^{\prime},\left|\sum_{i=2^{m-2}+1}^{2^{m-1}} x_{i}\right|^{\prime},\left|\sum_{i=2^{m-1}+1}^{3.2^{m-2}} x_{i}\right|^{\prime},\left|\sum_{i=3.2^{m-2}+1}^{2^{m}} x_{i}\right|^{\prime}\right) \\
& \leq \ldots \\
& \leq M^{m} \max _{1 \leq i \leq 2^{m}}\left|x_{i}\right|^{\prime} .
\end{aligned}
$$


Lemma 7. Let $\overline{\mathbf{Z}}$ be the image of $\mathbf{Z}$ in $R$.

For $n \in \mathbf{N}$,

$$
|| \bar{n}|||\leq 2 n||| 1|| \mid .
$$

Proof. We use the dyadic trick (Lemma 6) with $|.|^{\prime}=|\|||$.$\| and M=2$. Take $m=\left[\log _{2} n\right]+1, n \leq 2^{m} \leq 2 n$, and $x_{i}=1$ for $1 \leq i \leq n$. We have

$$
\left\||| n|||=||| \sum_{i=1}^{n} x_{i}\left|\| \leq 2^{m}\right||| 1|||\leq 2 n||| 1|| \mid .\right.
$$

Lemma 8. Let $\overline{\mathbf{Z}}$ be the image of $\mathbf{Z}$ in $R$.

For $n \in \mathbf{N}$,

$$
|\bar{n}| \leq n
$$

Proof. Using Lemma 7,

$$
\| \overline{n^{k}}|||=|\left|\bar{n}^{k}\right||| \leq 2 n^{k}|||1|||
$$

and $\left\|\left|\bar{n}^{k}\right|||^{1 / k} \leq 2^{1 / k} n|\| 1|||^{1 / k}\right.$. Taking $k \rightarrow+\infty$ we have $|\bar{n}| \leq n$.

Proposition 2. For $x, y \in R,|x+y| \leq|x|+|y|$.

Proof. We consider first the case when $R$ is a commutative ring with unit element. Let $x, y \in R$ and $n \geq 1$. Let $m=\left[\log _{2} n\right]+1$. Using Lemma 5 and Lemma 7 we have

$$
\begin{aligned}
\left|(x+y)^{n}\right| & =\left|\sum_{i=0}^{n}\left(\begin{array}{c}
n \\
i
\end{array}\right) x^{i} y^{n-i}\right| \\
& \leq\left(2 C_{1}^{2 \alpha}\right)^{m} \max _{0 \leq i \leq n}\left|\left(\begin{array}{c}
n \\
i
\end{array}\right) x^{i} y^{n-i}\right| .
\end{aligned}
$$

Now using Lemma 4 and Lemma 8,

$$
\begin{aligned}
\left|(x+y)^{n}\right| & \leq\left(2 C_{1}^{2 \alpha}\right)^{m} \max _{0 \leq i \leq n}\left|\left(\begin{array}{c}
n \\
i
\end{array}\right)\right||x|^{i}|y|^{n-i} \\
& \leq\left(2 C_{1}^{2 \alpha}\right)^{m} \max _{0 \leq i \leq n}\left(\begin{array}{c}
n \\
i
\end{array}\right)|x|^{i}|y|^{n-i} \\
& \leq\left(2 C_{1}^{2 \alpha}\right)^{m} \sum_{i=0}^{n}\left(\begin{array}{c}
n \\
i
\end{array}\right)|x|^{i}|y|^{n-i} \\
& \leq\left(2 C_{1}^{2 \alpha}\right)^{m}(|x|+|y|)^{n} .
\end{aligned}
$$

Finally exploiting the multiplicative property for $|$.$| ,$

$$
|x+y|=\left|(x+y)^{n}\right|^{1 / n} \leq\left(2 C_{1}^{2 \alpha}\right)^{\frac{1}{n}\left(\left[\log _{2} n\right]+1\right)}(|x|+|y|),
$$

and passing to the limit $n \rightarrow+\infty$ we get the sharp triangle inequality $|x+y| \leq$ $|x|+|y|$. We observe that the better bound obtained using the "dyadic trick" is crucial in this last step.

In the case where $R$ is a division ring we can assume that $x \neq 0$ and we obtain in the same way

$$
\left|\left(1+x^{-1} y\right)^{n}\right| \leq\left(2 C_{1}^{2 \alpha}\right)^{m}\left(1+|x|^{-1}|y|\right)^{n} .
$$


So multiplying by $|x|^{n}$ and using the multiplicative property we prove the same inequality as before. The end of the proof is identical.

\section{REFERENCES}

[Bou] N. Bourbaki, Commutative algebra Elements of Mathematics, chapter VI, section 6.1, Addison-Wesley, 1972. MR 50:12997

[Os] A. Ostrowski, Über einige Lösungen der Funktionalgleichung $\varphi(x) \cdot \varphi(y)=\varphi(x y)$ Acta Mathematica, 41, 1917, p. 271-284.

[We] D. Welsh, Codes and cryptography Oxford Science Publications, 1988. MR 89i:94001

Department of Mathematics, University of California, Los Angeles, California 90024

E-mail address: munoz@math.ucla.edu 